\title{
Análise de redes sociais de clientes com acometimento renal crônico em hemodiálise
}

\author{
Social network analysis of clients with chronic renal failure on hemodialysis \\ Análisis de redes sociales de clientes con insuficiencia renal crónica en hemodiálisis
}

Recebido: 05/11/2021 | Revisado: 15/11/2021 | Aceito: 15/11/2021 | Publicado: 17/11/2021

\author{
Izabella Andrade da Rocha \\ ORCID: https://orcid.org/0000-0002-9121-0972 \\ Universidade do Estado do Rio de Janeiro, Brasil \\ E-mail: iza_belbella@hotmail.com \\ Rafael Abrantes de Lima \\ ORCID: https://orcid.org/0000-0002-3843-7297 \\ Universidade Federal do Estado do Rio de Janeiro, Brasil \\ E-mail: rafael.abrantes83@gmail.com \\ Helena Maria Scherlowski Leal David \\ ORCID: https://orcid.org/0000-0001-8002-6830 \\ Universidade do Estado do Rio de Janeiro, Brasil \\ E-mail: helenalealdavid@gmail.com
}

\begin{abstract}
Resumo
Objetivo: caracterizar as redes de relações e interações dos clientes com acometimentos renais crônicos em hemodiálise e descrever as relações com os profissionais de saúde. Método: trata-se de um estudo descritivo e exploratório, com abordagem quantitativa de análise de redes sociais, o cenário de pesquisa um hospital público universitário do estado do Rio de Janeiro. Os sujeitos da pesquisa foram pessoas acometidas pela DRC em fase terminal. O estudo foi preconizado Resolução Número 510, de 07/04/2016. Resultados: foi observado prevalência do sexo masculino, média de idade de 48 anos, prevalecendo os aposentados, com renda entre 1 a 2 salários-mínimos, $85 \%$ sem vínculo empregatício, prevalecendo a raça parda, ensino médio completo predominou, são casados e média de 6 anos de diálise. Conclusão: por fim, foi possível analisar, identificar, descrever e discutir as redes sociais em como se configuram e seu papel na contribuição frente à adesão ao tratamento.

Palavras-chave: Insuficiência renal crônica; Diálise renal; Rede social; Cooperação e adesão ao tratamento; Enfermagem.
\end{abstract}

\begin{abstract}
Objective: to characterize the networks of relationships and interactions of clients with chronic kidney disease on hemodialysis and describe their relationships with health professionals. Method: this is a descriptive and exploratory study, with a quantitative approach of social network analysis, the research setting a public university hospital in the state of Rio de Janeiro. The research subjects were people affected by CKD in terminal phase. The study was recommended Resolution Number 510, of 04/07/2016. Results: it was observed prevalence of male gender, average age of 48 years, prevailing retired people, with income between 1 to 2 minimum wages, $85 \%$ without employment relationship, prevalence of brown race, complete high school predominated, are married and average of 6 years of dialysis. Conclusion: Finally, it was possible to analyze, identify, describe and discuss the social networks as they are configured and their role in contributing to treatment adherence.
\end{abstract}

Keywords: Chronic renal failure; Renal dialysis; Social network; Cooperation and adherence to treatment; Nursing.

\section{Resumen}

Objetivo: caracterizar las redes de relaciones e interacciones de los clientes con acometidos renales crónicos en hemodiálisis y describir las relaciones con los profesionales de la salud. Método: se trata de un estudio descriptivo y exploratorio, con abordaje cuantitativo de análisis de redes sociales, en el centro de investigación de un hospital público universitario del estado de Río de Janeiro. Los sujetos de la investigación eran personas afectadas por la ERC en fase terminal. El estudio fue recomendado Resolución Número 510 del 07/04/2016. Resultados: se observó prevalencia del sexo masculino, edad promedio de 48 años, predominio de jubilados, con ingresos entre 1 a 2 salarios mínimos, $85 \%$ sin relación laboral, predominio de la raza morena, predominio de la enseñanza media completa, son casados y promedio de 6 años de diálisis. Conclusión: Por último, fue posible analizar, identificar, descifrar y discutir las redes sociales en su configuración y su papel en la contribución frente a la adhesión al tratamiento.

Palabras clave: Insuficiencia renal crónica; Diálisis renal; Red social; Cooperación y adherencia al tratamiento; Enfermería. 


\section{Introdução}

O termo Rede Sociais (RS) possui variadas interpretações de acordo com cada área de estudo sob a forma de redes de empresas, de políticas, de movimentos sociais, dentre outros. Tal fato ocorre devido aos múltiplos fatores que atuam de forma simultânea gerando uma nova realidade social (David et al., 2018; Junqueira et al., 2015; Lanzoni \& Meirelles, 2012).

As redes podem ser entendidas como a forma de visualizar o indivíduo e seus atributos, para as relações que eles estabelecem entre si. Além de enfocar o vínculo interpessoal, a rede também considera os recursos e simbólicos que os grupos dispõem para compor a dinâmica da vida, seja em um grupo primário, como a família ou um grupo secundário, como as associações formais (Silva, Moura \& Junqueira, 2015).

Além disso, a rede como uma realidade social pode criar conhecimentos que lhes são próprios, numa perspectiva intersetorial e que resulta das relações internas e externas às organizações (Azevedo, 2016; Silva, Moura \& Junqueira, 2015).

Entretanto, existem relatos de equipes que constroem "uma rede de apoio interno" que parece ser fundamental para compor um "todo mais forte", que ultrapassa a pessoa, criando um corpo institucional. Além disso, possibilita o aprendizado dos trabalhadores e a criação de questionamentos sobre o fazer profissional, criando mecanismos de cuidado e vínculo com os usuários (Maximino, 2017).

A relação entre profissional e cliente deve ocorrer à aceitação do outro, a empatia, a relação de confiança e provavelmente a ausência de julgamentos, o que deve despertar a consciência dos clientes, do que sentem e do que precisam (Lucena et al., 2018).

Com base nisso, uma boa relação entre os clientes com acometimentos renais crônicos e profissionais de saúde é fundamental, pois a Hemodiálise (HD) é uma terapia renal substitutiva (TRS) exaustiva por ser três vezes na semana com uma duração de quatro horas por dia, o que torna o processo desgastante, tanto para o paciente quanto para o profissional de saúde (Ponce et al., 2019).

As DRCs são alterações que afetam tanto a estrutura quanto a função renal, com múltiplas causas e múltiplos fatores de risco. Trata-se de uma doença insidiosa, progressiva, irreversível, tornando-se perceptível quando seu estadiamento é na fase terminal, necessitando assim entrar em terapia renal substitutiva (Jesus et al., 2019).

A Sociedade Brasileira de Nefrologia (SBN), no último censo brasileiro de diálise referente a década 2009-2018 que o número estimado de pacientes em diálise é de 133.464 pacientes o que corresponderia a taxas de prevalência e incidência por milhão de população de 640 e 204 respectivamente (Neves et al., 2020).

Portanto, nesse estágio os clientes podem apresentar distúrbios emocionais tristeza, melancolia e podendo chegar a quadros depressivos, estando diretamente relacionado seu bem-estar à adesão e importância na evolução da saúde desses indivíduos em tratamento (Pretto et al., 2020; Alavarez, Rosendo \& Alchieri, 2016).

Portanto, vale ressaltar a importância da expansão das RS como método facilitador dos processos de cuidado ao cliente com acometimento renal crônico. A relação entre profissionais de saúde e pacientes garantindo que é uma interação humana e que possui mecanismos mentais utilizados no processo de desenvolvimento da doença (Pretto et al., 2020).

A importância da manutenção do equilíbrio emocional, motivação e o apoio na união da experiência da enfermidade e tratamento cria situações que reduzem a tensão e auxiliam na adaptação ao processo da doença. Nesse sentido, as redes utilizadas por usuários e profissionais tem como base o compartilhamento de objetivos, sentimentos, propósitos e confiança (Alavarez, Rosendo \& Alchieri, 2016).

Portanto, é de total importância evidenciar estudos de redes sociais, uma vez que as relações interpessoais podem modificar a prática assistencial prestada pelos profissionais de saúde aos pacientes renais crônicos. O objeto deste estudo delimitou-se a configuração das redes sociais entre clientes com acometimentos renais crônicos e profissionais de saúde.

O objetivo dessa revisão foi caracterizar as redes de relações e interações dos clientes com acometimentos renais 
crônicos em hemodiálise e descrever as relações com os profissionais de saúde. Como as redes sociais de clientes com acometimentos renais se organizam (ou configuram)? Assim justifica-se por ser uma temática pouco explorada principalmente no âmbito da saúde, e por acreditar que a análise de redes sociais em saúde permite ferramentas que são ricas e úteis para compreensão das relações entre profissionais da saúde e clientes.

\section{Metodologia}

Trata-se de um estudo descritivo e exploratório, com abordagem quantitativa de análise de redes sociais. A análise de rede social (ARS) visa mapear as interações entre atores, dando ênfase para as medidas e fluxos de informações entre eles. A análise permite "mensurar os modelos de relacionamento, bem como as inter-relações dos atores em configuração de rede, com base em seus contatos (Minayo \& Sanches, 1993; Borgatti, Everett \& Johnson, 2013; David et al., 2018; Junqueira et al., 2015; Silva, Moura \& Junqueira, 2015; Lanzoni \& Meirelles, 2012).

Teve como cenário de pesquisa um hospital público universitário do estado do Rio de Janeiro que possui serviço de nefrologia, mais especificamente salão próprio para realização de hemodiálise em clientes com acometimentos renais crônicos.

Os sujeitos da pesquisa foram pessoas acometidas pela DRC em fase terminal que é caracterizado por clientes com a Taxa de Filtração Glomerular $<15 \mathrm{ml} / \mathrm{min} / 1,73 \mathrm{~m}^{2}$ e que possuem acesso vascular pérvio que permita a realização da terapia renal substitutiva. Os critérios de inclusão para participação na pesquisa foi, pacientes acima de um ano de tratamento hemodialítico e maiores de 18 anos. E como critério de exclusão para usuários do serviço foi clientes que foram submetidos ao transplante, clientes em tratamento conservador, clientes em diálise peritoneal ou que por algum motivo esteja impossibilitado de participar da pesquisa.

As siglas utilizadas a partir daqui para identificação são: E clientes foi utilizado o shape up triangle na cor verde, TÉC para técnicos de enfermagem foi utilizado o shape diamond na cor amarela, ENF para enfermeiros foi utilizado o shape circle na cor azul, MÉD para médicos foi utilizado o shape square na cor laranja, SERV. SOC. para serviço social foi utilizado o shape plus (+) na cor preto, PSI para psicologia foi utilizado o shape down triangle na cor rosa, NUTRI para nutrição foi utilizado o shape box (quadrado com sinal de + interno) na cor verde e aqueles que apresentarem um " * a ao lado da sigla, significa que o profissional foi citado, porém não foi entrevistado.

Os dados foram extraídos através de uma entrevista individual em campo, realizada em dezembro de 2019 utilizando um roteiro semi-estruturada com o propósito de ser um subsídio para a análise diagnostica das redes sociais de pessoas tratadas pela hemodiálise. Inicialmente os dados foram inseridos no Excel e no Word com a finalidade de analisar o material obtido no trabalho de campo, descrevendo e analisando as configurações das redes sociais de clientes renais crônicos e profissionais de saúde.

No seguimento quantitativo, a análise se deu através da metodologia de Análise de Redes Sociais. Com os dados, foi possível mensurar, analisar e retratar a posição dos atores para, posteriormente, compreender a rede, seus nós e suas relações. Para o aprofundamento da análise das redes sociais, após as respostas em que os participantes citaram os profissionais que mais ajudavam na adesão aos cuidados, assim os achados foram inseridos em uma planilha utilizando Excel, após foi desenvolvida uma matriz utilizando esta planilha diretamente no software Ucinet $\odot$ e Netdraw $\odot$, permitindo assim avaliar as medidas de densidade da rede, graus de centralidade e intermediação, uma vez que esse método oferece métricas para análise de redes.

Chama-se sociograma a representação da RS em forma gráfica, incidindo em um tipo de retrato de como os atores sociais se relacionam e se agrupam. A representação se faz por pontos e linhas; os pontos representando os elos, "nós" (atores). As linhas (arestas) representam a ligação entre os nós da rede (David et al., 2018; Junqueira et al., 2015; Silva, Moura \& Junqueira, 2015; Lanzoni \& Meirelles, 2012). 
O estudo foi preconizado na Resolução No 196, de 10 de outubro de 1996, na Resolução No 466 de 12 de dezembro de 2012 e na Resolução № 510, de 07 de abril de 2016, ou seja, o projeto de pesquisa foi previamente submetido ao Comitê de Ética e Pesquisa da Universidade do Estado do Rio de Janeiro, via Plataforma Brasil, mediante carta de anuência e aprovado, sob o CAAE: 26288519.4.0000.5259 e parecer de número 3.766.472.

\section{Resultados}

A Tabela 1 mostra as principais características sociodemográficas da população de clientes estudados $(n=13)$. Foi observado sete $(54 \%)$ clientes do sexo masculino, com média de 48 anos de idade, 11 (85\%) são aposentados, oito (62\%) possuem renda entre 1 a 2 salários-mínimos, 11 (85\%) não possui vínculo empregatício, sete (54\%) se descrevem da raça parda, 7 (54\%) possuem o ensino médio completo, cinco (38\%) são casados e têm média de 6 anos de diálise.

Tabela 1. Características sociodemográficas dos clientes atendidos com doença renal crônica em um hospital universitário público estadual do Rio de Janeiro, RJ, Brasil, 2020.

\begin{tabular}{|c|c|c|}
\hline VARIÁVEL & $\mathbf{N}$ & $\%$ \\
\hline \multicolumn{3}{|l|}{ Sexo } \\
\hline Masculino & 7 & 53,8 \\
\hline Feminino & 6 & 46,2 \\
\hline \multicolumn{3}{|l|}{ Faixa Etária } \\
\hline 18 a 30 anos & 4 & 30,8 \\
\hline 31 a 45 anos & 2 & 15,3 \\
\hline 46 a 60 anos & 3 & 23,1 \\
\hline Mais de 60 anos & 4 & 30,8 \\
\hline \multicolumn{3}{|l|}{ Ocupação } \\
\hline Aposentado & 11 & 84,6 \\
\hline Comerciante & 1 & 7,7 \\
\hline Cozinheiro & 1 & 7,7 \\
\hline \multicolumn{3}{|l|}{ Renda } \\
\hline 1 a 2 salários mínimos & 8 & 61,5 \\
\hline 3 a 4 salários mínimos & 4 & 30,8 \\
\hline 5 ou mais salários mínimos & 1 & 7,7 \\
\hline \multicolumn{3}{|l|}{ Vínculo Empregatício } \\
\hline Não & 11 & 84,6 \\
\hline Sim & 2 & 15,4 \\
\hline \multicolumn{3}{|l|}{ Raça/Cor } \\
\hline Parda & 7 & 53,8 \\
\hline Preta & 3 & 23,1 \\
\hline Branca & 3 & 23,1 \\
\hline \multicolumn{3}{|l|}{ Escolaridade } \\
\hline Ensino Fundamental Incompleto & 1 & 7,7 \\
\hline Ensino Médio Incompleto & 2 & 15,4 \\
\hline Ensino Médio Completo & 7 & 53,8 \\
\hline Ensino Superior Incompleto & 2 & 15,4 \\
\hline Ensino Superior Completo & 1 & 7,7 \\
\hline
\end{tabular}




\begin{tabular}{ccc} 
Situação Conjugal & & \\
Casado & 5 & 38,4 \\
Divorciado & 2 & 15,4 \\
Viúvo & 2 & 15,4 \\
Solteiro & 4 & 30,8 \\
Tempo de Tratamento & & \\
1 a 5 anos & 6 & 46,2 \\
6 a 10 anos & 7 & 53,8 \\
\hline
\end{tabular}

Fonte: Autores (2020).

Neste primeiro sociograma, Gráfico 1, a pergunta de disparo foi aplicada para os clientes, totalizando 13 entrevistados, os clientes citaram até 5 profissionais que mais lhe ajudam na adesão ao cuidado, um dado importante é que nem todos os citados foram entrevistados, os não entrevistados têm uns asteriscos para facilitar na interpretação dos dados.

A rede de clientes versus profissionais possui 29 nós, 45 arestas de 812 Relações Possíveis, 46 Relações Existentes, com densidade de 5,7\%. A densidade avalia a ligação dos nós dentro de uma rede e assim percebemos que pelo valor, é possível inferir que a densidade da rede é baixa ou, de acordo com a ARS, que a rede tem conexão frágil.

Portanto, os atores com alta centralidade de grau são aqueles que aparecem com maior número de contatos diretos com clientes renais crônicos, são os que aparecem no Gráfico 2 com maior tamanho em relação aos demais nós da rede. Nesta rede destaca-se as devidas importâncias assistenciais aos atores técnicos de enfermagem e enfermeiros, podendo relacionar ao acompanhamento, atenção e o modo de agir durante as sessões de hemodiálise.

\section{Discussão}

Os resultados obtidos no estudo demonstram uma concentração de $(53,8 \%)$ de homens, na faixa etária entre 18 e 30 anos ou acima de 60 anos. Na década 2009-2018, onde foi realizado o Censo Brasileiro de Diálise demonstrou uma população majoritariamente masculina (58\%) em fase adulta que segundo o estudo pode ser explicado pelo aumento da expectativa de vida da população em um modo geral (Neves et al., 2020). Diversos estudos desenvolvidos com pessoas que realizam hemodiálise no Brasil também referem este predomínio do sexo masculino em fase adulta (Moreira \& Borges, 2020; Jesus et al., 2019; Marinho et al., 2017; Bastos \& Kirsztajn, 2011).

Relacionando o aumento da expectativa de vida de clientes hemodialíticos pode-se explicar quanto o manejo clínico em realizar um diagnóstico precoce, fazendo com que o cliente tenha melhor qualidade de vida e assim possa tratar o quanto antes, aumentando assim relação com idades entre 18 e mais de 60 anos de idade (Jesus et al., 20149).

Com relação à raça, observou-se um maior número de pessoas que se auto declararam pardas (53,8\%) (Santana et al., 2019). Estes dados corroboram com o estudo no qual sinaliza um aumento significativo na proporção de casos entre indivíduos autodeclarados como pardos em relação aos demais indivíduos (Bousquet-Santos, Costa \& Andrade, 2019; Melo, Melo \& Vilar, 2018).

Bem como, a ocupação dos clientes em hemodiálise pudemos identificar que (84,6\%) estão aposentados pois é uma prerrogativa do Instituto Nacional de Seguridade Social em seu artigo 523, item 2, onde inclui-se a nefropatia grave e pode ser observado em estudos que tem como população os clientes renais crônicos hemodialítico (Carvalho et al., 2020; Pretto et al., 2020; Silva \& Mattos, 2019). 
Na sequência, discorreu-se sobre o nível de escolaridade dos clientes entrevistados, onde (53,8\%) possuem o ensino médio completo e que é necessário que os clientes tenham condições de entender, significar e aplicar as instruções que recebem, sendo corroborado outro estudo de mesmo impacto (Rocha \& Figueiredo, 2019; Rocha \& Pinho, 2019).

Contudo, em relação ao tratamento hemodialítico, alinhar estudos, com o tempo de tratamento obtido neste estudo de 6 a 10 anos (53,8\%) e de 1 a 5 anos (46,2\%), que não houve grande distribuição percentual e foi onde observamos ser um ponto atípico, pois estudo semelhante, apontou tempo de tratamento hemodialítico variando entre 3 e 4 anos (Santana, et al., 2019).

Quanto à situação conjugal, a distribuição percentual também não variou em grandes proporções, porém, ainda é mais evidente o número de pessoas casadas $(38,4 \%)$, e isto nos aponta que "em pesquisa qualitativa sobre a percepção do conforto em clientes de hemodiálise foi evidenciado que existem efeitos positivos decorrentes da presença de cônjuge, familiares e amigos" (Melo et al., 2019). Abaixo, o sociograma de análise estrutural da rede clientes desta pesquisa, Gráfico 1.

Gráfico 1. Rede Social dos Clientes. Rio de Janeiro, RJ, Brasil, 2020

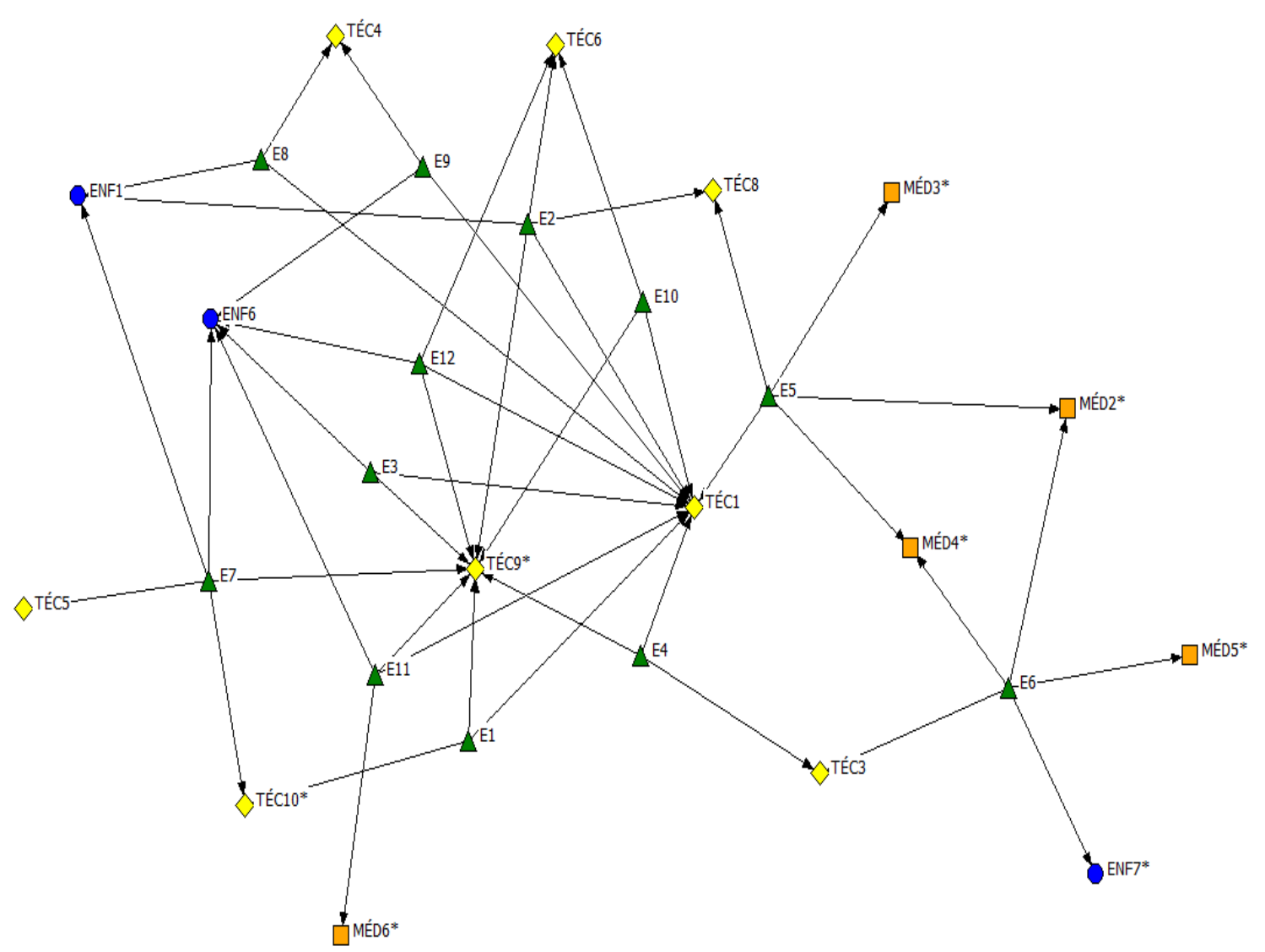

Fonte: Autores (2020).

Para exemplificar melhor a discussão da RS, se traçarmos um comparativo de que uma alta densidade em seu limite máximo de 100\% fossem sem atores, os 5\% encontrados aqui seriam respectivamente cinco atores (Fonseca, 2017).

$\mathrm{Na}$ ARS as pessoas com relacionamentos mais distantes, possuem ligações fracas e estão envolvidas em menor grau. Tais ligações fracas são as responsáveis pela baixa densidade em uma rede, onde muitas possibilidades de relacionamentos estão ausentes (Silva, 2019). 
Assim, nem todos os atores da rede estão em contato direto uns com os outros, trocando informação ou qualquer tipo de recurso, o que torna a rede pouco coesa. As redes mais conectadas, por sua vez, possuem muitas ligações e, quanto maior for a medida de densidade, maior a coesão do grupo (Azevedo, 2016).

As relações entre os membros da equipe exercem influência na resolubilidade das práticas de saúde (David et al., 2018; Junqueira et al., 2015; Lanzoni \& Meirelles, 2012). Nesse sentido, RS tecidas entre usuários e profissionais de saúde, pode significar e representar uma maior adesão às atividades coletivas de promoção da saúde, e um fortalecimento das práticas ofertadas ou desenvolvidas no território de saúde, induzindo uma apropriação política das relações comunitárias para a formação de novas redes sociais ou para o incremento da participação nas redes já existentes (Lucena et al., 2018).

Assim o cuidado é um processo que acontece através do diálogo entre os atores dos serviços de saúde. Baseia-se na comunicação e estabelece elementos que podem evidenciar códigos, valores, símbolos e normas (Feuerwerker, 2014; Lima, Moreira \& Jorge, 2013).

A centralidade é definida pelo número de contatos diretos que um ator possui com os demais elos da rede, este sendo um canal direto de informação (David et al., 2018; Junqueira et al., 2015; Silva, Moura \& Junqueira, 2015; Lanzoni \& Meirelles, 2012). Abaixo, o sociograma referente a centralidade de grau desta rede, Gráfico 2.

Gráfico 2. Rede social dos clientes tratados por hemodiálise: centralidade de grau. Rio de Janeiro, RJ, Brasil, 2020

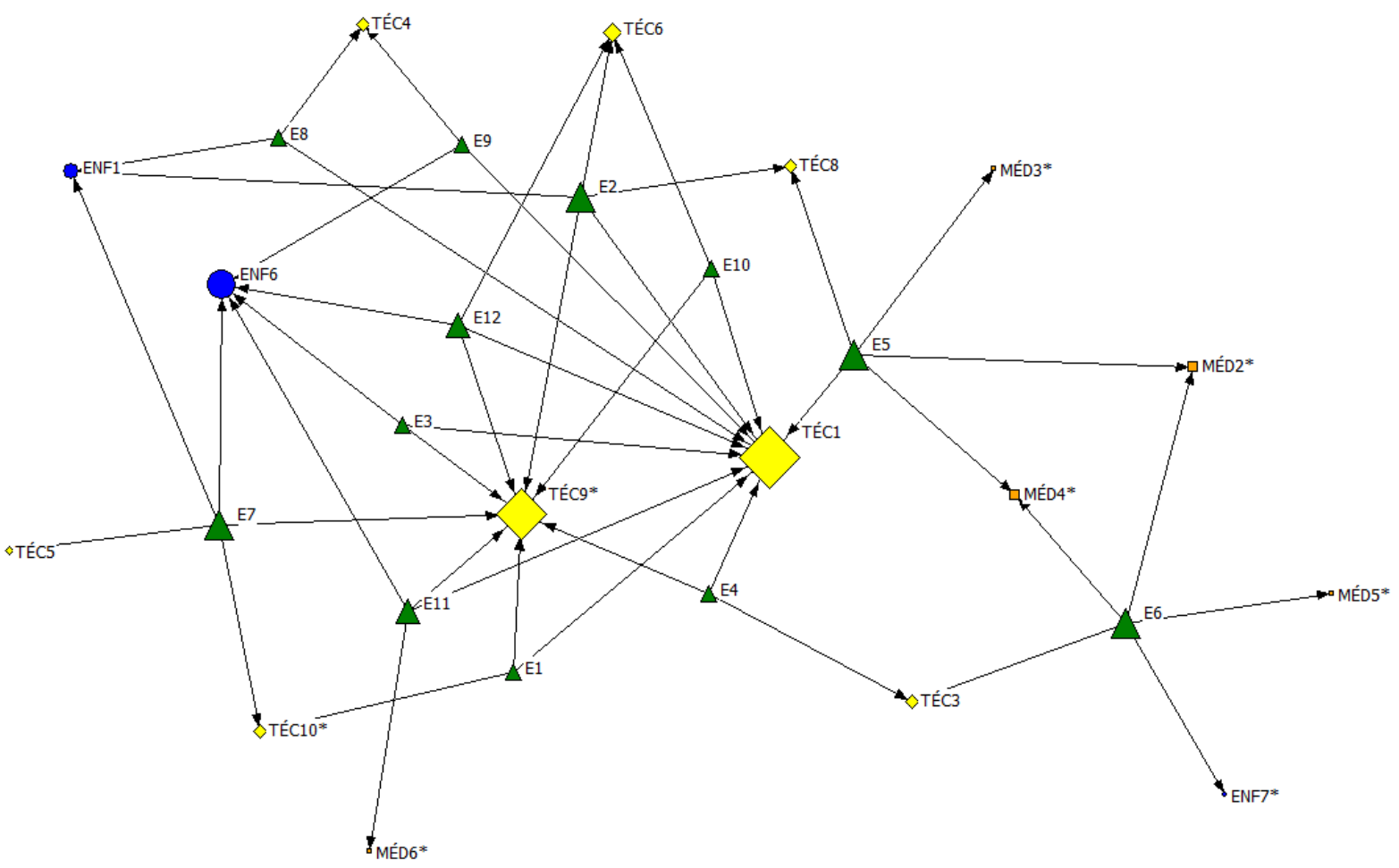

Fonte: Autores (2020).

Percebemos que alguns técnicos de enfermagem e médicos possuem seus graus de centralidade atribuídos às falas durante as transcrições das entrevistas, e podemos correlacionar com o fator de quem mais presta assistência e a influência que exercem no seu tratamento uma vez que pode ser influenciado pela dinâmica de serviço.

Enquanto a análise da posição dos atores na rede percebemos que dois técnicos de enfermagem e um enfermeiro TÉC1, TÉC9* e ENF6, concentram o maior número de entrada de setas, o que mostra maior número de contato com os 
clientes detendo maior centralidade na rede, isso pode se dar por serem profissionais de maior escuta ativa uma vez que são os profissionais da enfermagem que estão mais próximos e por mais tempo durante as sessões de hemodiálise.

Tendo a dinâmica do serviço descrita, detalhamos que o corpo da enfermagem circula no salão a todo o tempo, realizando os cuidados diretos aos clientes que vai desde o controle do peso de início e saída da terapia, cálculo do peso interdialítico, aferição de sinais vitais, administração de medicamentos seja de rotina ou de intercorrências, realização de curativo, intervindo nas intercorrências, bem como também prestando aconselhamento e efetivando humanização a essa população, mas um fator importante também a ser considerado é a característica dos pacientes dos turnos que são diferentes.

A população de clientes do turno da manhã e da tarde são mais acordados, com maior interação com a equipe, demandando mais atenção dos profissionais. Entretanto, os clientes do terceiro turno em sua maioria são pacientes internados seja por intercorrências no percurso do tratamento, seja por urgência dialítica, seja por questões de doenças de base e que em sua maioria encontram-se mais debilitados e não tendem a interagir tanto.

O papel desses profissionais na rede é de orientação e com o poder de articulação que possuem, acabam sendo veículos de cuidado através de educação em saúde, gerando menos ansiedade e maior proximidade, ou seja, estreitando relações, criando vínculos e consequentemente criando redes, mesmo em um cenário que foge ao padrão da atenção básica.

Nesta mesma rede identificamos a posição do TÉC5 totalmente isolado, sem ter laços com os outros indivíduos pertencentes a rede, isso nos faz questionar o porquê de tal localização dentro da rede, podemos então confrontar com a fala do próprio profissional na entrevista.

"Difícil ter essa relação social entre cliente e profissional, eu acho um meio das pessoas não trocarem os papéis e esquecer que você é um profissional" (TÉC5).

Podemos perceber também que o serviço de psicologia não foi citado pelos clientes como atores que geram influência, que tenham alguma importância ou que tenham construído algum tipo de vínculo com cliente e assim contribuindo para uma melhor adesão ou quiçá até como rede de apoio. No salão de hemodiálise a psicologia dá suporte aos pacientes, atende por demanda o que pode justificar esse achado.

O profissional MÉD1, SERV. SOC, ENF2, ENF3 e ENF5 que foram entrevistados, não são citados e não entra no sociograma, vendo esta informação, recorreu às entrevistas e pôde-se perceber que em nenhum momento o profissional cita quando é perguntado sobre o seu papel no atendimento aos clientes é percebido e como que eles integram a rede social do serviço.

O profissional NUTRI1 também não é citado e não entra no sociograma, a partir daí buscou-se entender o que acontece e recorro novamente as entrevistas, sendo nesta situação há uma diferença dos profissionais supracitados, onde o profissional diz quando questionado se os clientes percebem o seu papel no atendimento prestado:

"[...]até os próprios clientes tem mês que eu venho converso com eles e não sinto muito interesse deles e tem esse retorno assim, mas também quando não tem nenhum retorno eles ficam mais chateados". (NUTRI1).

O que causa estranheza, pois a fala do profissional vai ao encontro do que pode ser evidenciado e podemos questionar se realmente este serviço se faz ser entendido e procura de alguma forma integrar-se à rede social do cliente.

O profissional ENF4 não é citado e, também não compõem o sociograma, retornando as entrevistas percebo a seguinte fala da profissional:

"Tem uns que tem o vínculo mais próximo mesmo, de amizade com a gente e outros veem só como se fosse nosso papel a mão de obra e só, que tem que fazer aquilo ali e pronto" (ENF4).

O que leva ao questionamento de que, se a fala contraditória entre ser percebida como parte integrante de uma rede social com a fala de que por outros ela é vista apenas como mão de obra. 
O fato do profissional TÉC7 não ser citado e não compor o sociograma permeia por uma questão ambígua durante a sua entrevista, na qual foi falada em duas partes. Quando questionado como que os clientes a percebem em relação a sua assistência houve o seguinte comentário:

"Bom meu papel é colocar o cliente na máquina, instalar ele na máquina, tomar conta enquanto eles dialisam[...]". (TÉC7).

Em contrapartida quando questionado como ela integra a rede social a seguinte fala emerge:

"[...] devido ao tempo que eles ficam aqui se tratando 3 vezes na semana durante 4 horas, não tem como não criar um vínculo a maioria vem aqui e trata a gente como família mesmo" (TÉC7).

É questionável como estes profissionais realmente se fazem perceber e se fazem presentes a uma assistência holística que visa entender o cliente como um todo e integrar a rede social e por consequência a adesão deste cliente.

O TÉC2 também não é citado e nem entra no sociograma pois deixa claro que não possui nenhum tipo de relação com os clientes ao dizer:

\section{"[... ]eu não posso responder porque eu não tenho esse vínculo com eles" (TÉC2).}

Será o seu papel enquanto profissional de saúde, meramente tecnicista? Ou seja, chega ao plantão, trabalha e vai embora, sendo característico do modelo biomédico.

Porquanto, observo que o cliente E13 não referenciou nenhum profissional de saúde não sendo visível sua participação no sociograma, para tanto recorro as entrevistas e deparo com a seguinte fala:

"Olha, aqui já teve algumas pessoas que ajudavam, hoje em dia "tá" mais, as pessoas estão mais dispersas, não dá aquela atenção que dava entendeu? Mas não tem, outro jeito a gente vai levando" (E13).

Esse modelo biomédico ainda amplamente trabalhado em nosso país, onde o foco do trabalhador é no procedimento, no corpo é identificado uma queixa, na tentativa de transformá-la em um possível diagnóstico. Com isso, a queixa deixa de fazer parte da pessoa e se transforma em um sintoma, uma doença (David et al., 2018; Melo, Melo \& Vilar, 2018; Junqueira et al., 2015).

O serviço de hemodiálise tem essa característica bastante presente por ser um serviço especializado que requer uma tecnologia dura, máquinas que fazem hoje a função renal, a técnica e os equipamentos tem a centralidade no trabalho em diálise, isso pode ser a explicação para este modelo encontrado, o que é um grande desafio, encontrar estratégias de tornar esse cuidado hoje centralizado no procedimento em um cuidar individualizado.

Este modelo ainda é bastante presente nos serviços de saúde, é preciso mudar a ótica dos profissionais para uma pratica assistencial com foco para o sujeito de forma complexa que abrange não só o aspecto biológico ou ausência de doença, mas também o bem-estar físico, mental, social. Para que assim possa se pensar no cuidado humanizado, ainda é preciso quebrar paradigmas quanto a esse modelo enraizado (Junqueira et al., 2015).

Sob a ótica da humanização fala-se de um cuidado que visa a integralidade do sujeito que implicará no seu acolhimento, na sua escuta, no vinculo e na relação estabelecida, visto a crescente complexidade do sistema de saúde e que deva dar mais ênfase na criação de ambientes menos alienantes que valorizem a dignidade do usuário (Goulart \& Chiar, 2010).

Na condição de assistência com humanização há problemas que dificultam esse processo, mas que também se trata do que cada profissional espera de sua profissão, onde que para alguns é apenas um dever a ser cumprido em contrapartida já aqueles que querem realmente fazer a diferença na vida do próximo (Goulart \& Chiar, 2010).

Estes clientes têm suas particularidades e suas dificuldades se tratando de uma doença cônica, que torna a vida deste indivíduo restritiva a muitos aspectos que compromete, além do aspecto físico, o psicológico, com repercussões pessoais, familiares e sociais. 
O acompanhamento por equipe interdisciplinar se justifica pela complexidade da DRC, que compreende diferentes facetas em seu tratamento e incorpora múltiplos campos de conhecimento, incluindo aspectos relacionados à condição clínica, nutricional, emocional e de adesão à terapia medicamentosa (Alvarez, Rosendo \& Alchieri, 2016).

Desse modo a multidisciplinaridade associado a integralidade do cuidado tem que emergir como condição fundamental por ser tratar de habilidades que exigem dos profissionais envolvidos com este público, atitude como disciplina, escuta ativa percepção e compreensão, empatia para a partir deste ponto traçar cuidados (Santos et al., 2019).

Sendo uma enfermidade com uma evolução longa, dolorosa e traumática, exige uma equipe interdisciplinar treinada para prestar assistência total e que priorize a comunicação entre enfermeiros, nefrologistas, nutricionistas, assistentes sociais e psicólogos (Alvarez, Rosendo \& Alchieri, 2016).

Chama atenção ainda a cliente E6, é possível extrair do sociograma a demanda de profissionais que está cliente procura, podemos levantar algumas questões que pode ser o motivo para tal necessidade. Na sua fala:

"Eles cuidam, tiram dúvidas, me ensinam sobre a doença, muita coisa, eu mesmo fazendo diálise há 5 anos tem coisa que eu ainda estou descobrindo[...]" (E6).

Essa fala demonstra a necessidade de conhecimento sobre a doença, essa cliente tem pouco tempo de TRS, isso pode levar a uma necessidade maior de conhecimento, como também mobiliza mais profissionais porque ainda não se estabeleceu seus vínculos.

No tocante a entrada do paciente em terapia hemodialítica, informações extremamente específicas inerentes ao tratamento devem ser fornecidas previamente e não no momento que o paciente já está em sala para realizar a terapia ou em urgência dialítica (Lins et al., 2018).

A orientação em linguagem adequada das diferentes complicações da DRC e suas possíveis consequências aumenta a aderência ao tratamento, a explicação ilustrada com réplicas de alimentos facilita o entendimento e motiva o seguimento dietético, a demonstração sobre as técnicas de aplicação de medicamentos diminui os erros de administração, o apoio psicológico ameniza o impacto da doença, a identificação e o aconselhamento sobre questões socioeconômicas constituem aspectos importantes, os quais, sem dúvida, podem ter impactos decisivos para uma melhor evolução da doença renal crônica (Melo et al., 2019; Santos et al., 2019).

Devemos destacar que o indivíduo deva ser orientado sobre a doença, o seu tratamento, riscos e benefícios associados a hemodiálise, bem como acessos vasculares, dietas, terapias medicamentosas e rigorosa adesão ao tratamento para que ele possa ter melhor qualidade de vida (Santos et al., 2019).

No Quadro 1, seguem as medidas de centralidade de grau dos atores desta rede, separando os graus, nesta pesquisa como os clientes relacionam-se apenas com profissionais e não entre si, apresentação de grau será somente grau de saída, tornando-se um viés. 
Quadro 1. Medidas de centralidade de grau (clientes). Rio de Janeiro, RJ, Brasil, 2020

\begin{tabular}{|c|c|}
\hline ATORES & GRAU DE SAÍDA \\
\hline E1 & 3 \\
\hline E2 & 5 \\
\hline E3 & 3 \\
\hline E4 & 3 \\
\hline E5 & 5 \\
\hline E6 & 5 \\
\hline E7 & 5 \\
\hline E8 & 3 \\
\hline E9 & 3 \\
\hline E10 & 3 \\
\hline E11 & 3 \\
\hline E12 & 4 \\
\hline E13 & 0 \\
\hline
\end{tabular}

Fonte: Autores (2020).

\section{Limitações do estudo}

No Quadro 1 quando relacionado as centralidades de grau, os atores (pacientes vs pacientes) não ocorreu a interação a nível de grau de entrada entre os mesmos, pois não foi o objetivo do estudo.

\section{Considerações Finais}

Ao longo da pesquisa, analisando as redes sociais dos clientes renais crônicos podemos perceber uma baixa densidade, ou seja, nos demonstra uma fragilidade no vínculo criado entre os atores.

Conforme foi delimitado a pesquisa que não relacionou a interação entre os próprios clientes tornou-se um viés na centralidade de grau, pois não foi possível analisar o grau de entrada. Por fim, foi possível analisar, identificar, descrever e discutir as redes sociais em como se configuram e seu papel na contribuição frente à adesão ao tratamento.

Porém, foi visualizado também elos fracos no que diz respeito aos cuidados dos clientes renais crônicos, com relação às categorias profissionais de serviço social, nutrição e psicologia e que há abertura e possibilidade de melhora neste aspecto.

Sendo assim, sugere-se a realização de novos estudos futuros com a temática principal do estudo e principalmente com relação rede social entre paciente vs paciente e paciente vs profissional como método de cuidados em saúde, com vista a qualidade de vida dos pacientes nefropatas; a inclusão da temática na formação do profissional enfermeiro a nível stricto sensu e que, contudo, revela a necessidade do aprofundamento nos conteúdos de nefrologia. 


\section{Referências}

Alvarez, P. E. S., Rosendo, E., \& Alchieri, J. C. (2016). The applicability of the concept of treatment adherence in the context of the Brazilian mental health system. Rev. Esc. Enferm. USP, 50(spe), 54-60. http://dx.doi.org/10.1590/S0080-623420160000300008.

Azevedo, B. M. S. (2016). A aposta no encontro para a produção de redes de produção de saúde (Tese de Doutorado). Universidade Estadual de Campinas, Campina, SP, Brasil. http://repositorio.unicamp.br/jspui/bitstream/REPOSIP/313068/1/Azevedo_BrunoMarianideSouza_D.pdf.

Bastos, M. G., \& Kirsztajn, G. M. (2011). Doença renal crônica: importância do diagnóstico precoce, encaminhamento imediato e abordagem interdisciplinar estruturada para melhora do desfecho em pacientes ainda não submetidos à diálise. J. bras. nefrol, 33(1), 93-108. https://doi.org/10.1590/S010128002011000100013

Borgatti, S. P.; Everett, M. G.; \& Johnson, J. C. (2013). Analyzing social networks. 1 ed. Califórnia: Sage Publications.

Bousquet-Santos, K., Costa, L. G., \& Andrade, J. M. L. (2019). Estado nutricional de portadores de doença renal crônica em hemodiálise no Sistema Único de Saúde. Ciênc. Saúde Colet, 24(3), 1189-1199. https://doi.org/10.1590/1413-81232018243.11192017.

Carvalho, L., Cesarino, C. B., Garcia, L. F., Ribeiro, R. H., Orlandi, F. S., \& Kusumota, L. (2020). Efeitos da doença e adequação da hemodiálise em pacientes com rigidez arterial. Esc. Anna Nery Rev. Enferm, 24(2), e20190068. https://doi.org/10.1590/2177-9465-EAN-2019-0068.

David, H. M. S. C., Faria, M. G. A., Dias, J. A. A., Silva, T. F., Souza, V. M. D., \& Dias, R. S. (2018). Análise de redes sociais na atenção primária em saúde: revisão integrativa. Acta Paul. Enferm, 31(1), 108-115. https://doi.org/10.1590/1982-0194201800016.

Feuerwerker, L. C. M. (2014). Micropolítica e saúde: produção do cuidado, gestão e formação. Porto Alegre: Rede UNIDA.

Fonseca, J. S. A. (2017) Redes sociais na regulação da assistência à saúde em um município de pequeno porte do Rio de Janeiro (Mestrado em enfermagem). Universidade Estadual do Rio de Janeiro, Rio de Janeiro, RJ, Brasil.

Goulart, B. N. G., \& Chiar, B. M. (2010). Humanização das práticas do profissional de saúde - contribuições para reflexão. Ciênc. Saúde Colet, 15(1), 255268. https://doi.org/10.1590/S1413-81232010000100031.

Jesus, N. M., Souza, G. F., Mendes-Rodrigues, C., Almeida Neto, O. P., Rodrigues, D. D. M., \& Cunha, C. M. (2019). Qualidade de vida de indivíduos com doença renal crônica em tratamento dialítico. J. bras. Nefrol, 41(3), 364-374. https://doi.org/10.1590/2175-8239-JBN-2018-0152.

Junqueira, L. A. P., Campos, M. A., Silva, M. F., \& Barbosa, R. P. (2015). Redes sociais e relações de poder na enfermagem: estudo de caso em um hospital municipal. Rev. Fac. Cienc. Med. Sorocaba, 17(3), 148-158. https://revistas.pucsp.br/index.php/RFCMS/article/view/24413/pdf.

Lanzoni, G. M. M., \& Meirelles, B. H. S. (2012). A rede de relações e interações da equipe de saúde na Atenção Básica e implicações para a enfermagem. Acta Paul. Enferm, 25(3), 464-470. https://doi.org/10.1590/S0103-21002012000300023.

Lima, L. L., Moreira, T. M. M., \& Jorge, M. S. B. (2013). Produção do cuidado a pessoas com hipertensão arterial: acolhimento, vínculo e corresponsabilização. Rev. bras. Enferm, 66(4), 514-522. https://doi.org/10.1590/S0034-71672013000400008.

Lins, S. M. S. B., Leite, J. L., Godoy, S., Tavares, J. M. A. B., Rocha, R. G., \& Silva, F. V. C. (2018). Adesão de portadores de doença renal crônica em hemodiálise ao tratamento estabelecido. Acta Paul. Enferm, 31(1), 54-60. https://doi.org/10.1590/1982-0194201800009.

Lucena, L. N., Rodrigues, M. P., Melo, R. H. V., Andrade, F. B., Vilar, R. L. A., \& Filho, J. B. (2018). Avaliação da satisfação do usuário com o acolhimento na estratégia da saúde da família no Recife (PE). Rev. Ciênc. Plur, 4(2), 21-37. https://periodicos.ufrn.br/rcp/article/view/16837.

Marinho, A. W. G. B., Penha, A. P., Silva, M. T., \& Galvão, T. F. (2017). Prevalência de doença renal crônica em adultos no Brasil: revisão sistemática da literatura. Cad. saúde colet, 25(3), 379-388. https://doi.org/10.1590/1414-462X201700030134.

Maximino, V. S., Liberman, F., Frutuoso, M. F., \& Mendes, R. (2017). Profissionais como produtores de redes: tramas e conexões no cuidado em saúde. Saúde soc, 26(2), 435-447. https://doi.org/10.1590/S0104-12902017170017.

Melo, G. A. A., Aguiar, L. L., Silva, R. A., Quirino, G. S., Pinheiro, A. K. B., \& Caetano, J. A. (2019). Fatores relacionados ao conforto prejudicado em pacientes renais crônicos hemodialíticos. Rev. Bras. Enferm, 72(4), 889-895. https://doi.org/10.1590/0034-7167-2018-0120.

Melo, R. H. V., Melo, M. L. , \& Vilar, R. L. A. (2018). Análise de redes sociais: a reciprocidade entre usuários e profissionais na estratégia saúde da família. Rev. Ciênc. Plur, 4(1), 22-35. https://periodicos.ufrn.br/rcp/article/view/13626.

Minayo, M. C. S.; \& Sanches, O. (1993). Quantitativo-Qualitativo: Oposição ou Complementaridade? Cad. Saúde Publ., 9(3), p. 239-262.

https://www.scielo.br/pdf/csp/v9n3/02.pdf.

Moreira, R. A., \& Borges, M. S. (2020). Perfil e nível de esperança entre pacientes que realizam hemodiálise e diálise peritoneal. Cogitare enferm, 25 , e67355. http://dx.doi.org/10.5380/ce.v25i0.67355.

Neves, P. D. M. M., Sesso, R. C. C., Thomé, F. S., Lugon, J. R., \& Nascimento, M. M. (2020). Censo Brasileiro de Diálise: análise de dados da década 20092018. J. Bras. Nefrol, 42(2), 191-200. https://doi.org/10.1590/2175-8239-JBN-2019-0234.

Ponce, K. L. P., Tejada-Tayabas, L. M., González, Y. C., Haro, O. H., Zúñiaga, M. L., \& Morán, A. C. A. (2019). Nursing care for renal patients on hemodialysis: challenges, dilemmas and satisfactions. Rev. Esc. Enferm. USP, 53, e03502. https://doi.org/10.1590/S1980-220X2018011103502.

Pretto, C. R., Rosa, M. B. C., Dezordi, C. M., Benetti, S. A. W., Colet, C. F., \& Stumm, E. M. F. (2020). Depressão e pacientes renais crônicos em hemodiálise: fatores associados. Rev. Bras. Enferm, 73(Suppl1):20190167. https://doi.org/10.1590/0034-7167-2019-0167. 
Research, Society and Development, v. 10, n. 15, e223101522867, 2021

(CC BY 4.0) | ISSN 2525-3409 | DOI: http://dx.doi.org/10.33448/rsd-v10i15.22867

Rocha, K. T., \& Figueiredo, A. E. (2019). Letramento em Saúde: Avaliação de pacientes em terapia renal substitutiva. Enferm. nefrol, 22(4), 388-397. http://dx.doi.org/10.4321/s2254-28842019000400005.

Rocha, R. P. F., \& Pinho, D. L. M. (2019). Ocorrência de eventos adversos em unidades públicas de hemodiálise. Enferm. glob, 18(3), 1-34. https://doi.org/10.6018/eglobal.18.3.343361.

Santos, B. T. U., Araújo, S. T. C., Torres, D. G., Bastos, S. S. F., Azevedo, A. L., \& Neves, K. C. (2019). Integralidade do cuidado-permanência na nefrologia: concepções de enfermeiros. Rev. Esc. Enferm. USP, 53, e03523. https://doi.org/10.1590/S1980-220X2018043703523.

Santana, E. C., Silva, M. S. C., Silva, T. R. G., Oliveira, A. D. S., Ribeiro, I. P., \& Madeira, M. Z. A. (2019). Perfil dos pacientes submetidos a tratamento hemodialítico em uma clínica em Teresina. Rev. Pesqui, 11(1), 142-146. http://dx.doi.org/10.9789/2175-5361.2019.v11i1.142-146.

Silva, M. F., Moura, L. R., \& Junqueira, L. A. P. (2015). As Interfaces entre Empreendedorismo Social, Negócios Sociais e Redes Sociais no Campo Social. Revista de Ciências da Administração, 17(42), 121-130. https://doi.org/10.5007/2175-8077.2015v17n42p121.

Silva, P. E. B. B., \& Mattos, M. (2019). Complicações hemodialíticas na unidade de terapia intensiva. Rev. enferm. UFPE on line, 13(1), 162-168. https://doi.org/10.5205/1981-8963-v13i01a234781p162-168-2019.

Silva, T. F. (2019). Acolhimento, redes sociais e produção de cuidado (Tese de Doutorado). Universidade do Estado do Rio de Janeiro, Rio de Janeiro, RJ, Brasil. 